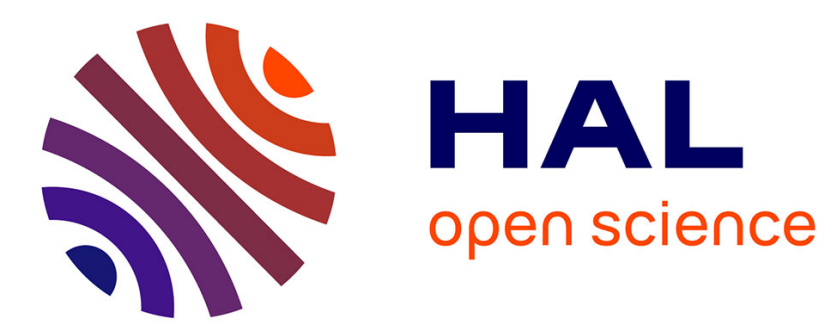

\title{
Représenter les citoyens via les groupes d'intérêts
}

Emmanuelle Reungoat

\section{To cite this version:}

Emmanuelle Reungoat. Représenter les citoyens via les groupes d'intérêts: enjeux et lacunes d'un système communautaire routinisé. Savoir / Agir , 2013, 26, pp.103-110. 10.3917/sava.026.0103 . hal-02892320

\section{HAL Id: hal-02892320 \\ https://hal.science/hal-02892320}

Submitted on 7 Jul 2020

HAL is a multi-disciplinary open access archive for the deposit and dissemination of scientific research documents, whether they are published or not. The documents may come from teaching and research institutions in France or abroad, or from public or private research centers.
L'archive ouverte pluridisciplinaire HAL, est destinée au dépôt et à la diffusion de documents scientifiques de niveau recherche, publiés ou non, émanant des établissements d'enseignement et de recherche français ou étrangers, des laboratoires publics ou privés. 


\section{Europe}

\section{Représenter les citoyens via les groupes d'intérêts}

\section{Enjeux et lacunes \\ d'un système communautaire routinisé}

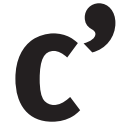

est à la fois pour répondre au diagnostic de "déficit démocratique» du système représentatif (la majorité des citoyens européens s'abstiennent lors de l'élection européenne depuis 1999) et au besoin d'expertise des administrations de l'Union européenne qu'a été promue la pratique, aujourd'hui solidement ancrée, de l'association des groupes d'intérêt au processus décisionnel européen. En proposant une analyse critique de ce dispositif de gouvernement, on mettra en avant, sur la base des apports des analyses empiriques, les lacunes d'un système progressivement élevé au rang de modèle démocratique alternatif permettant l'association continue de la "société civile» au processus de décision. Il s'agira, également, en questionnant son adéquation à un régime démocratique, d'esquisser quelques pistes de réflexion et d'évoquer les revendications existantes à cet égard ${ }^{\mathrm{T}}$.

Fortement intégrés de longue date au système politique et promus depuis une dizaine d'années par les textes communautaires comme les représentants de la

I. Cet article actualise certains aspects du dossier paru sous le titre «Un peuple européen sur mesure » coordonné par Hélène Michel dans Savoirlagir, n ${ }^{\circ}$, mars 2009. On pourra notamment reliredans ce dossier : Hélène Michel, « "Société civile" ou peuple européen ? L'Union européenne à la recherche d'une légitimité politique».

\author{
EmmanuelLe Reungoat \\ ATER à l'Institut d'études politiques \\ de Strasbourg
}

«société civile» européenne (Traité de Lisbonne, II art. 8B.2), notion à juste titre très discutée, les groupes d'intérêt sont présents à tous les stades de la préparation d'une décision. Ils interviennent auprès de ses différentes instances: Parlement, Conseil et Commission notamment, cette dernière ayant largement œuvré à leur institutionnalisation. En général, leur intégration dans les processus de décision trouve ses fondements théoriques non dans la démocratie représentative, mais dans les principes du pluralisme ${ }^{2}$. On renvoie également, au niveau communautaire, à la promotion de la démocratie dite délibérative.

La dimension conflictuelle des intérêts sociaux en compétition, fondatrice dans les théories pluralistes américaines en particulier, semble cependant gommée au niveau européen par l'insistance sur la recherche du consensus via des

2. Pour une critique de l'idéologie pluraliste en jeu dans la représentation des intérêts et de l'expertise, $C f$. H. Michel et C. Robert (dir)., La fabrique des "Européens", Strasbourg, PUS, 2010. 
procédures de délibération et l'indifférenciation des types d'intérêts à représenter. Tendant à une dépolitisation des enjeux, le système politique européen promeut une "société civile » traversée d'intérêts distincts et néanmoins conciliables. À distance d'une conception du politique comme mode de reconnaissance et de règlement des conflits, le développement d'une culture du consensus, liée pour partie aux origines technocratiques d'un projet européen construit à côté et en opposition à la politique partisane et parlementaire, tend à négliger les rapports de force inhérents à la gestion des affaires publiques. En privilégiant ce nouveau type de participation et son autorégulation, la configuration institutionnelle et la culture politique qui se développent à Bruxelles tendent à mettre à distance certains registres d'expression politique (en particulier contestataire), voire certains groupes, en particulier les citoyens les moins dotés.

\section{Egalité formelle, déséquilibre réel}

Deux apports majeurs issus des études empiriques prenant pour objet les pratiques de représentation des groupes d'intérêt peuvent être soulignés: tous les intérêts ne peuvent faire l'objet d'une représentation auprès des institutions et quand c'est le cas, les groupes ne peuvent assurer celle-ci avec un égal pouvoir d'influence. Basée sur un pied d'égalité formel, la participation des groupes d'intérêt au processus décisionnel n'est pondérée ni en fonction d'une capacité « quantitative » de représentation (liée aux effectifs des organisations), ni en fonction de la teneur des intérêts défendus ou des caractéristiques des populations représentées. L'autorégulation des groupes d'intérêt prônée avec constance par la Commission depuis les années 1990 conduit à de fortes disparités de présence. Leur regroupement sous une appellation unique ne doit pas masquer des différences très importantes entre ces acteurs. Deux types de groupes peuvent notamment être distingués à partir de la nature des intérêts défendus, que ceux-ci soient privés et économiques, ou publics, ne servant pas les intérêts exclusifs de leurs membres. Si l'écart semble s'être réduit dans la décennie 2000 , les groupes représentant des intérêts privés et économiques demeurent majoritaires à Bruxelles. En 2002 , ils étaient $67 \%$ contre $23,4 \%$ agissant pour des intérêts "publics» s'appuyant sur la défense d'une cause d'intérêt général. Aujourd'hui ces derniers représenteraient près du tiers des groupes d'intérêt européens ${ }^{3}$, contrairement à ce que le label de représentants de la «société civile » pourrait laisser entendre. De nombreux intérêts restent donc peu représentés ou absents.

On retrouve ici un talon d'Achille classique de nombreuses théories pluralistes qui négligent les coûts de l'auto-organisation pour certaines populations. Loin d'être automatique, la structuration en groupe de pression peut se révéler d'autant plus difficile que l'intérêt est collectif ou diffus. Ce qui se vérifie largement au niveau européen. Ainsi lorsque des groupes défendant des intérêts publics sont actifs et insérés au niveau communautaire, c'est souvent à l'initiative des institutions elles-mêmes et via leurs financements. En faisant l'impasse à la fois sur les coûts de l'action collective et sur les inégalités sociales face à la délibération, l'institutionnalisation de cette représentation n'assure pas une forte expression de la

3. Ces chiffres sont à considérer avec prudence au vu des difficultés de recension des groupes d'intérêts. $C f$. J. Greenwood, Interest Representation in the European Union, Basingstoke, Palgrave, 20 I I. 
diversité des perspectives défendues dans la représentation de la population européenne au risque d'exclure pour partie les groupes les moins dotés.

L'association des groupes d'intérêt dans le processus décisionnel peut parfois également dépendre de leur alignement général au point de vue des institutions. Ainsi les groupes dont les objectifs sont globalement contraires à la logique de l'intégration libérale des marchés peuvent parfois se voir exclus du processus et contraints de réinterpréter leur corps de valeurs. ${ }^{4}$ Par ailleurs, la dépendance économique peut tendre à renforcer une logique de «féodalisation». Ainsi la fidélité de l'activité de représentation peut s'avérer conditionnée par les moyens financiers à la disposition des représentants. Dans les années 1990 et 2000, afin d'obtenir des fonds européens et être reconnues partenaires, certaines organisations non gouvernementales ont pu tendre à s'éloigner de leurs objectifs originaux, au risque de se transformer parfois en «sous-traitants» des priorités intergouvernementales. Pour prendre un exemple, si l'organisation Fundación Secretariado Gitano en Espagne profite d'une forte croissance dans les années 2000 en obtenant l'aide européenne, la hiérarchie de ses objectifs (visible dans l'évolution des budgets) change. La prévalence conférée à la lutte contre le chômage sur la promotion de l'éducation épouse les priorités de l'Union européenne. Ce faisant, l'objectif initial d'aide à une intégration plus égalitaire des populations gitanes est mis en question. Si les gitans trouvent des emplois grâce au programme, ceux-ci

4. Voir par exemple, R. Eising, The Political Economy of State-Business Relations in Europe, London, Routledge, 2009. C. Woll, «Trade Policy Lobbying in the European Union ", in D. Coen \& J. Richardson (eds), Lobbying the European Union, 2009, Oxford University Press, p. 277-297. sont peu qualifiés. Leur exclusion semble se perpétuer quand un programme d'éducation pouvait ambitionner de faciliter leur accès à des études universitaires, ainsi qu'à un meilleur niveau d'emploi et statut social.s

\section{Expertise et jeux d'influence}

Outre cet écart de présence, les représentants des citoyens organisés n'accèdent pas à d'égales capacités d'influence et de participation dans le processus décisionnel. Plusieurs études s'accordent à montrer que ce sont les groupes disposant de ressources économiques et humaines importantes, d'expérience à l'international, de réseau social et de capital culturel, qui sont les plus à même de faire entendre leurs intérêts auprès des institutions de l'Union européenne. Les organisations ayant les moyens financiers d'entretenir un siège à Bruxelles et une équipe de permanents incluant des consultants, autrement dit un personnel d'experts formé aux procédures techniques et culturelles de l'Union européenne (formation juridique, maîtrise de plusieurs langues, connaissance des usages informels des institutions), s'ouvrent un meilleur accès et influent plus fortement sur le processus décisionnel que les autres. La consultation ouverte dans la dernière décennie pour la définition de la politique climatique de l'Union après 20I2, concernant en particulier la régulation des émissions de gaz à effet de serre, en constitue un exemple.

Pour A.T. Gullberg qui l'analyse, si la transparence du processus est assurée, celui-ci n'est inclusif qu'« en surface» et marqué par «une représentation disproportionnée » dans la mesure où, dans

5. R. Sanchez-Salgado, Comment l'Europe construit la société civile, Paris, Dalloz, 2007, p. 519-520. 
chacun des volets de la consultation, les lobbyistes issus de l'industrie dominent largement la représentation grâce à des ressources beaucoup plus importantes et mieux adaptées que les celles des organisations non gouvernementales. ${ }^{6}$ C'est vrai tant au niveau des contributions produites sur invitation de la Commission en 2004 (78 documents de positionnement soumis pour les organisations des entreprises et industries contre 30 pour les organisations environnementales) qu'en termes de capacité à envoyer des représentants lors des sessions de consultation organisées en 20062007 sur l'enjeu décisif de la mise en place $\mathrm{du}$ « système communautaire d'échange de quotas d'émissions » (SCEQE) permettant de définir les secteurs à inclure et les règles d'allocation des permis d'émissions. Quand l'ensemble des organisations non gouvernementales environnementales envoie cinq à six membres par session, à elle seule, la Key Stakebolder alliance for ETS Review regroupant les partisans d'une révision de la SCEQE et constituée par les organisations des industries à forte intensité énergétique, est capable de mandater entre huit et douze représentants à chaque session, sans compter les représentants d'autres groupes d'intérêt industriels. Au contraire de leurs homologues, les représentants des industries sont également capables de rester présents auprès des différentes directions et institutions sur l'ensemble de la procédure de codécision. Cette « asymétrie de voix et d'influence laisse des traces sur les résultats politiques ». L'obtention, par les industries à haute intensité énergétique, d'une exception au principe de la mise aux enchères totale des quotas d'émission en porte la trace.

6. Cf. Transparency International, Global Corruption Report : Climate Change, 20I0, p. 43-48. http ://www.transparency.org/whatwedo/ pub/global_corruption_report_climate_change
Nombreux sont les exemples qui donnent à voir comment, par leurs ressources, les groupes représentant des intérêts économiques dominent largement ce mode de représentation, même si en leur sein, les disparités de moyens peuvent créer de fortes inégalités entre les pays membres. Ceux-ci sont également favorisés par l'antériorité de leur présence à Bruxelles. Ce déséquilibre de la représentation des intérêts au sein de l'Union européenne ne lui est pas spécifique et constitue une faiblesse classique des systèmes pluralistes. Le phénomène est néanmoins renforcé par la diffusion du recours à l'expertise comme répertoire légitime d'action collective à Bruxelles, qui contribue largement à la professionnalisation des lobbyistes, y compris au sein des groupes défenseurs d'intérêts publics, et par la difficulté renforcée, pour les citoyens ordinaires, de participer au régime par rapport aux échelles nationales.

\section{Représentation et démocratie européenne}

L'association des groupes d'intérêt au processus de décision et l'élection des députés au parlement européen constituent les deux uniques modes de représentation directe des citoyens à l'échelle de l'Union européenne. Or, on sait que tout acte de représentation, y compris au sein d'une démocratie représentative, n'est ni également ni nécessairement démocratique. Le régime démocratique peut être caractérisé sur la base de trois principes fondamentaux, transversaux à ses différentes définitions: il s'agit d'un système garantissant à la fois le pouvoir du peuple, dont il tient sa légitimité, ainsi que l'égalité politique des citoyens traduite par une égale influence de chacun sur le gouvernement. L'Union européenne s'inscrit dans 
cette optique (selon le traité de Lisbonne, «l'Union respecte le principe de l'égalité de ses citoyens, qui bénéficient d'une égale attention de ses institutions, organes et organismes ", II art. 8A). Enfin, la démocratie assure que chacun ait un contrôle sur le processus de décision dans la mesure où celle-ci est soumise au contrôle populaire.

L'institutionnalisation de l'association des groupes d'intérêt à la prise de décision peine à respecter ces réquisits. On notera tout d'abord que la capacité de figuration de l'ensemble de la société civile européenne par les groupes d'intérêt reste à la fois trop méconnue et controversée pour faire fonctionner un pacte symbolique de représentation $\mathrm{du}$ "peuple» (comme c'est le cas des parlements nationaux). En outre, tel qu'il s'exerce au niveau de l'Union européenne, ce dispositif de gouvernement répond de manière exclusivement formelle à l'exigence d'égalité d'influence des citoyens. En refusant d'établir des critères de représentativité, le dispositif échoue à garantir la diversité des groupes, donc un réel pluralisme, et à réguler la mise en place de dominations d'intérêts particuliers puissants sur le pouvoir. La neutralité du recours à l'expertise qui légitime souvent ce dispositif est d'ailleurs contredite par les études qui rappellent en général le caractère profondément politique de l'activité et soulignent que les recommandations émises par les experts à l'échelle de l'Union européenne peuvent tendre à favoriser les intérêts des acteurs privés. ${ }^{7}$

La responsabilité (accountability) et la soumission à un contrôle public de l'action des représentants de ces groupes n'est pas pleinement assurée. Les textes

7. Voir par exemple I. Bruno \& al., in "L'européanisation saisie par son instrumentation", in B. Palier, Y. Surel, L'Europe en action, Paris, L'Harmattan, 2007. réglementaires européens restent très peu contraignants à cet égard et ne permettent pas toujours de garantir la transparence et la publicisation de leurs activités. Cette absence de contrôle et de responsabilité contraste avec l'intensité que leur intervention dans la prise de décision peut atteindre. Leur action va en effet souvent au-delà de la simple consultation officielle. Siles représentants des groupes d'intérêt ne sont pas les décideurs, certains deviennent parfois partie prenante de la décision. Il n'est pas rare de voir des amendements rédigés par eux être votés au Parlement européen. Un rapport commandé par le groupe parlementaire des Verts/Alliance Libre Européenne en 2008 montre ainsi que sur deux projets concernant la régulation des gaz à effet de serre et des produits chimiques (système REACH), les amendements parlementaires sont identiques ou très similaires aux propositions émises par les groupes d'intérêt industriels et rédigés hors du parlement. Il précise que sur le projet $\mathrm{REACH}$, plus de $50 \%$ des amendements présentés par le groupe PPE-ED sont issus de groupes d'intérêt appartenant en majorité aux industries chimiques. ${ }^{8}$ Plusieurs rapports de la Cour des comptes jugeaient déjà, à la fin des années I990, parfois excessif et abusif le rôle joué par ces acteurs au sein de la Commission, au risque de porter « atteinte à son autorité et à son indépendance $» .9$

Plusieurs tendances spécifiques du système européen s'affirment donc. L'Union européenne promeut une association renforcée de la «société civile» via les

\footnotetext{
8. M. Craig, Lobbying at the European parliament - two legislative cases: F-Gas and REACH, avril 2008, http ://archive.greens-efa.eu/cms/default/ dokbin/232/232162.lobbying_at_the_european_ parliament@en.pdf.

9. JO n ${ }^{\circ} \mathrm{C} 035$ du 09/02/1999, p. I- 52, http ://admi. net/eur/loi/leg_euro/fr_399Y020201.html
} 
groupes d'intérêts au titre de dispositif démocratique. En second lieu, elle veut également porter une attention renforcée à leur prise en considération. Enfin, on sait que la difficulté d'accès au système politique pour les simples citoyens se trouve clairement renforcée à l'échelle de l'Union européenne. Le niveau européen accroît l'élitisme de gouvernement présent au niveau national et la difficulté à représenter les intérêts publics et ceux des populations les moins dotées au sein des arènes de pouvoir. Ces discordances du système politique de l'Union européenne aux principes démocratiques et à un système plus inclusif permettent de tracer quelques pistes de réflexion.

Si elle est peu conforme à la tradition française d'organisation du système politique, l'association des groupes d'intérêt à la prise de décision est une pratique ancrée, conçue comme légitime, pourvoyeuse d'expertises requises par les institutions et largement routinisée depuis des décennies à l'échelle communautaire. Plusieurs propositions visent à réguler cette pratique par la mise en place de check and balances définis par les institutions. La diversité des groupes d'intérêts présents au niveau communautaire est réelle. Dans la dernière décennie notamment, l'insertion de représentants d'intérêts publics a ainsi permis d'y porter des normes éthiques basées sur des préoccupations civiques et sociales venant concurrencer notamment les normes de marché. Le pluralisme reste limité cependant et le dispositif tend à l'invisibilisation de certaines populations. Des mécanismes de promotion de l'organisation des intérêts diffus, tout comme la réforme et le développement du soutien financier aux associations les plus faibles ont pu être évoqués à cet égard. Certaines organisations proposent également une pondération de l'insertion des groupes dans les processus de décision: selon leur représentativité quantitative notamment, ou dans une optique qualitative, en fonction des intérêts défendus, et/ou des caractéristiques des populations représentées. À titre comparatif, de tels dispositifs ont pu être développés dans une optique de discrimination positive, pour pallier les inégalités produites par les systèmes représentatifs (règle de parité, découpage de circonscriptions favorisant certaines populations en fonction de critères spécifiques, ethniques par exemple, comme cela a pu être le cas pour améliorer la représentation des populations noires aux ÉtatsUnis).

La «transparence » de l'association des groupes d'intérêt devient également un enjeu croissant. Il reflète une tendance contemporaine à l'exigence de mécanismes de contrôle plus dynamiques et continus, au-delà des échéances électorales. Des propositions visant à accroître le contrôle sont avancées de longue date par les associations ou dans les rapports parlementaires. ${ }^{1 \circ}$ Un débat s'est organisé autour du caractère obligatoire de l'enregistrement des groupes d'intérêt dans un registre public conditionnant l'accès et la circulation au sein des institutions. Ce qui reviendrait à inviter chacun de ces groupes à rendre publics son employeur, les montants et objets de dépenses pour la/ les causes défendues, voire pour certaines associations, à produire des comptes-rendus d'activité dont le contrôle pourrait être dévolu à une autorité publique indépendante (voir les propositions d'AlterEU : http ://www.alter-eu.org). D’autres principes de régulation sont évoqués : distinction entre les types d'intérêts, code

Io. Voire D. Chabanet, «Les enjeux de la codification des groupes d'intérêt au sein de l'Union européenne ", Revue Française de Science Politique, 2009, Vol. 59 (5), p. 997-I019. 
de conduite des lobbyistes plus précis et contraignant, développement de sanctions dissuasives à l'encontre des représentants d'intérêt ne respectant pas les règles, déclaration d'intérêts financiers par les députés et leurs collaborateurs ou encore réglementation des intergroupes, qui réunissent, de manière informelle, des parlementaires de différents groupes politiques et des experts issus de la "société civile» sur des thèmes spécifiques. Pour réduire les risques de conflit d'intérêt concernant les parlementaires ou les commissaires, l'association Alter-EU prône enfin une période de transition de trois ans interdisant le passage entre la Commission et une fonction dans le secteur privé liée au domaine de politique publique concerné et demande l'interdiction, pour les groupes d'intérêt, d'employer des eurodéputés ou leurs assistants.

Plus globalement, si la mise en place d'un « gouvernement d'élites » est également caractéristique des systèmes politiques nationaux et renvoie à une critique classique des systèmes représentatifs, le phénomène s'accentue au niveau européen. Les degrés de compétence et de politisation exigés pour entrer dans le jeu politique européen consolident les processus d'exclusion des citoyens les moins dotés. D’autres phénomènes renforcent le fort biais élitaire au sein du parlement et des institutions de l'Union européenne: outre le caractère socialement différencié d'un abstentionnisme spécifiquement élevé lors des élections, le faible niveau d'information disponible, le renforcement du poids des élites des partis au détriment des militants dans les prises de position européennes et la composition sociologique du parlement y contribuent. À une couverture médiatique plus lâche s'ajoutent également la faiblesse du suivi des activités parlementaires de la part des partis nationaux et la difficulté des mouvements sociaux à développer leurs répertoires d'action traditionnels à l'échelle de l'Union européenne. Deux types d'organisations qui facilitent, au niveau national, l'exercice du contrôle populaire.

On rejoint ainsi plusieurs auteurs qui invitent à repenser la place du conflit (face au consensus) dans la vie politique européenne. Autrement dit à politiser l'Union, pour rendre ses décisions plus lisibles et transmissibles (par les partis, militants, médias et tout un ensemble de traducteurs intermédiaires du politique) à l'ensemble des citoyens. La mise en avant, au niveau communautaire, du débat public et de l'existence de choix entre des options politiques distinctes peut constituer une voie vers une participation politique progressivement élargie des citoyens. La recherche, à différentes échelles, de modes d'organisation plus inclusifs de l'ensemble de la population constitue également une voie de réduction des déséquilibres entre les perspectives et intérêts exprimés au sein des modes de représentation existants. Et, si cela ne suffira pas à résoudre les déséquilibres institutionnels de la "démocratie de couloir » qu'est l'Union européenne, des dispositifs de participation plus directe pourraient également être développés: mise en place d'une initiative législative contraignante, réflexion sur les conditions de révocabilité des élus voire des commissaires, développement de la pratique des consultations, notamment référendaires. En France, celles-ci ont montré comment, quand les conditions de lisibilité et d'appropriation citoyenne des questions communautaires sont réunies, les enjeux européens peuvent mobiliser (de 57,2\% des inscrits lors des élections européennes de 2004 , l'abstention est ainsi descendue à $30,6 \%$ lors du référendum sur le TCE de $2005)$. 